\title{
PTPN2 is associated with Crohn's disease and its expression is regulated by NKX2-3
}

\author{
Wei Yu ${ }^{\mathrm{a}}$, John P. Hegarty ${ }^{\mathrm{a}}$, Arthur Berg ${ }^{\mathrm{b}}$, Ashley A. Kelly ${ }^{\mathrm{a}}$, Yunhua Wang ${ }^{\mathrm{a}}$, Lisa S. Poritz ${ }^{\mathrm{a}, \mathrm{c}}$, \\ Andre Franke ${ }^{\mathrm{d}}$, Stefan Schreiber ${ }^{\mathrm{d}}$, Walter A. Koltun ${ }^{\mathrm{a}, *}$ and Zhenwu Lin ${ }^{\mathrm{a}}$ \\ ${ }^{a}$ Department of Surgery, Pennsylvania State University, Hershey, PA, USA \\ ${ }^{\mathrm{b}}$ Department of Public Health Sciences, Center for Statistical Genetics, Pennsylvania State University, Hershey, \\ $P A, U S A$ \\ ${ }^{\mathrm{c}}$ Department of Cellular \& Molecular Physiology, Pennsylvania State University, Hershey, PA, USA \\ ${ }^{\mathrm{d}}$ Institute for Clinical Molecular Biology, Department of General Internal Medicine, Christian-Albrechts \\ University, Kiel, Germany
}

\begin{abstract}
PTPN2 is a risk gene for Crohn's disease (CD). We investigated whether PTPN2 genetic variants (rs2542151 and rs2542152) were associated with CD in a familial IBD registry. Both rs2542151 and rs2542152 are associated with CD, but not ulcerative colitis (UC). mRNA expression levels of PTPN2 were significantly increased in intestinal tissues $(p=0.0493)$, and nearly significantly increased in B cells $(p=0.0889)$ from CD patients, but not significantly altered in UC. cDNA microarray results found that PTPN2 was down-regulated by NKX2-3 knockdown in human cells. We confirmed this observation by RT-PCR analyses in NKX2-3 knockdown in B cells from IBD patients and human intestinal microvascular endothelial cells (HIMEC). In addition, we found that mRNA expression of another IBD-associated gene, NKX2-3, was increased in intestinal tissues and B cells from CD patients, but not significantly increased in UC patients. A positive correlation was observed between mRNA expression of PTPN2 and NKX2-3 in B cells and in intestinal tissues from both CD and UC patients. These results suggest that PTPN2 may have an important role in CD pathogenesis and may represent a potential diagnostic and therapeutic target for IBD.
\end{abstract}

Keywords: PTPN2, association, Crohn's disease, gene expression, NKX2-3

\section{Introduction}

Inflammatory bowel diseases (IBD) are chronic inflammatory disorders of the gastrointestinal tract, primarily comprised of Crohn's disease (CD) and ulcerative colitis (UC). Both genetic and immunological factors are involved in IBD pathogenesis [1]. The Wellcome Trust Case Control Consortium (WTCCC) genome-wide association study reported a strong association $\left(p=4.6 \times 10^{-8}\right)$ of the single nucleotide polymorphism (SNP) rs2542151, located $5.5 \mathrm{~kb}$ upstream

*Corresponding author: Walter A. Koltun, MD and Zhenwu Lin, PhD. Division of Colon \& Rectal Surgery. Mail code H137. The Milton S. Hershey Medical Center, Penn State College of Medicine, 500 University Drive, P.O. Box 850, Hershey, PA 17033, USA. Tel.: +1 717531 5164; Fax: +1 717531 0646; E-mail: wkoltun@ hmc.psu.edu. of the PTPN2 gene (protein tyrosine phosphatase, nonreceptor type 2), with CD [2]. This association has been replicated by several follow-up studies [3-5]; however, a functional role for PTPN2 in CD pathogenesis remains unclear.

The family of protein tyrosine phosphatases (PTPs) plays a critical role in regulating fundamental cellular signaling events, including cell proliferation, differentiation and survival [6]. PTPN2 is expressed in intestinal epithelial cells and helps to maintain barrier function. It is a key negative regulator of important immune mediators STAT1 and STAT3, as well as p38 and ERK1/2 phosphorylation [7]. Two recent studies have reported increased PTPN2 expression in CD patients $[8,9]$.

We undertook a genetic association replication study for PTPN2 in patients from a familial IBD registry from central Pennsylvania. Two SNPs were genotyped: rs2542151, showing strong association in the WTCCC 
study; and rs2542152, a follow-up SNP of the WTCCC study, which is located $2.5 \mathrm{~kb}$ upstream of PTPN2. We then examined mRNA expression levels of PTPN2 in B cell lines from the familial IBD registry and in intestinal tissues from IBD patients.

NKX2-3 (NK2 transcription factor related, locus 3) is an IBD-associated intestinal transcription factor [1, 2] with increased levels of mRNA expression in $C D$ patients [10]. Our cDNA microarray data showed that PTPN2 expression is down-regulated following knockdown of NKX2-3 in B cells from IBD patients [11,12] and human intestinal microvascular endothelial cells (HIMEC) [13]. As this indicated that PTPN2 gene expression might be regulated by NKX2-3, we compared PTPN2 expression levels with expression of NKX2-3 in IBD patients.

\section{Materials and methods}

\subsection{Establishment of $B$ cell lines}

Epstein-Barr virus (EBV) was obtained from supernatants of the cotton-top tamarin cell line B95-8 (ATCC, Manassas, VA). Using standard procedures, B cell lines were established for individual family members entered into our IBD patient registry $[10,11]$. B cell lines were cultured in RPMI-1640 medium containing $10 \%$ fetal bovine serum (FBS) at $37^{\circ} \mathrm{C}$ in an atmosphere of $5 \% \mathrm{CO}_{2}$.

\subsection{Patients and intestinal tissue samples}

Patients undergoing surgery at the Penn State Hershey Medical Center for a diagnosis of CD participated in this study. An informed consent was signed by the patients before surgery. Intestinal tissues were immediately submerged in RNA later (Ambion, CA, USA) at $4{ }^{\circ} \mathrm{C}$ overnight, then stored frozen at $-70^{\circ} \mathrm{C}$ until total RNA extraction.

\subsection{DNA extraction and PTPN2 genotyping}

DNA was extracted from B cell lines and intestinal tissues using QIAamp DNA Mini Kit (QIAGEN Sciences, MD, USA). SNP rs2542152 was selected by Dr. Stefan Schreiber group as follow-up SNP of the WTCCC study for PTPN2 gene genotyping [4]. rs2542152 is located $2.5 \mathrm{~kb}$ upstream of PTPN2 transcriptional start site. SNPs rs2542151 and rs2542152 genotyping was performed using two methods: SNPlex ${ }^{\mathrm{TM}}$
Genotyping System [4] and PCR-based RFLP (restriction fragment length polymorphism) $[14,15]$ as previously described. For SNPlex ${ }^{\mathrm{TM}}$, $100 \mathrm{ng}$ of genomic DNA was dried overnight in TwinTec 384-well plates (Eppendorf, Hamburg, Germany) at room temperature. The genotyping process was performed using SNPlex ${ }^{\mathrm{TM}}$ chemistry (Applied Biosystems, Foster City, USA) on an automated platform with TECAN Freedom EVO and 384-well TEMO liquid handling robots (TECAN, Männedorf, Switzerland). For the RFLP method, rs2542151 is a natural HhaI recognition site, and rs2542152 is a converted MfeI recognition site. A 185 bp fragment containing SNP rs2542151 using primers GTAGCCCCACTTCGCCAATG (forward) and GTGGAGCTACACGGTGGAAG (reverse); and a 133 bp fragment containing SNP rs2542152 using primers CAGCTGCAGCATTTCACGTGG (forward) and CCACTGCCCTATGCGGTCAATT (reverse) were amplified with the following PCR profile: $95^{\circ} \mathrm{C} 5 \mathrm{~min}, 32$ cycles of $95^{\circ} \mathrm{C} 30 \mathrm{sec}, 58^{\circ} \mathrm{C} 45 \mathrm{sec}$ and $72^{\circ} \mathrm{C} 45 \mathrm{sec}$, and a final extension at $72^{\circ} \mathrm{C} 5 \mathrm{~min}$. PCR products were digested with HhaI (for rs2542151) and MfeI (for rs2542152) (New England Biolabs, Ipswich, MA), separated on 3\% agarose gel and visualized by ethidium bromide staining.

\subsection{Transfection and infection}

Small hairpin RNA (shRNA) vector targeting human NKX2-3 was generated using the pSUPER vector system as described previously $[11,16]$. The 19-nucleotide sequence within NKX2-3 targeted by the shRNA oligonucleotide pair was 5'-AGGAACATGAAGAGG AGCC-3'. Forward and reverse primers were annealed and ligated into the pSUPER.retro.puro vector (OligoEngine, WA, USA).

pSUPER.retro.puro.shRNA-NKX2-3 and empty vector were transfected into Phoenix-Ampho cells with Lipofectamine Plus (Invitrogen, CA, USA). Forty-eight hours posttransfection, $\mathrm{B}$ cells from one CD patient and one UC patient were infected with the viral supernatant. Forty-eight hours later, the infected cells were switched into a medium containing $0.4 \mu \mathrm{g} / \mathrm{ml}$ puromycin to start selection.

pSUPER.retro.puro.shRNA-NKX2-3 and empty vector were transfected into HIMEC with TransPass ${ }^{\text {TM }}$ HUVEC transfection reagent (New England BioLabs, USA) according to the manufacturer's instructions. Forty-eight hours posttransfection, mRNA from was isolated from the transfected HIMEC. 


\subsection{Microarray and data analysis}

Illumina Human HT12 v 3 Expression BeadChips (Illumina, CA) were used in this study. The BeadChip targets $>25,000$ genes with $>48,000$ probes. Microarray hybridization, data collection, and analysis were performed at the Genomics Core of the Cleveland Clinic Lerner Research Institute as described before [11].

\subsection{RNA extraction and reverse transcription-polymerase chain reaction (RT-PCR)}

Total RNA was extracted from EBV transformed B cell lines and intestine tissues using RNeasy mini Kit according the manufacturer's instructions [11] (QIAGEN Sciences, MD, USA). cDNA was synthesized from $1.5 \mu \mathrm{g}$ of total RNA using Superscript III $1^{\text {st }}$ Strand Synthesis Kit (Invitrogen, CA, USA).

Primers for human NKX2-3 (Forward 5' - CCACCCCTTTCTCAGTCAAA -3' and Reverse 5'- CTGCGG CTAGTGAGTTCAAA-3'), PTPN2 (Forward 5' - GGTGCAGCAGATGAAACAGA -3' and Reverse 5' - CAG TTTGGGGTTCAGAGGAA-3'), and GAPDH (Forward 5'-TGATGACATCAAGAAGGTGGTGAAG-3' and Reverse 5' -TCCTTGGAGGCCATGTGGGCCAT3') were designed using Primer3 software. PCR amplifications were performed at $94^{\circ} \mathrm{C}$ for 30 seconds, $58^{\circ} \mathrm{C}$ for 45 seconds, and $72^{\circ} \mathrm{C}$ for 45 seconds for 35 cycles (NKX2-3 and PTPN2) and 24 cycles (GAPDH) respectively, followed by final extension at $72^{\circ} \mathrm{C}$ for $7 \mathrm{~min}$. The PCR product for NKX2-3 is $210 \mathrm{bp}$, PTPN2 is $354 \mathrm{bp}$, and GAPDH is $236 \mathrm{bp}$. PCR products were visualized on $2 \%$ agarose gels stained with ethidium bromide.

\subsection{Western blotting}

Western blotting was performed as described previously [10]. Briefly, B cells and HIMEC were lysed with RIPA buffer (25 mM Tris-HCl, PH 7.6, $150 \mathrm{mM} \mathrm{NaCl,}$ $1 \%$ NP-40, $1 \%$ sodium deoxycholate, $0.1 \%$ SDS ) and protease inhibitor cocktail (Roche, Germany). Protein (30 $\mu \mathrm{g} /$ lane) was separated using SDS-15\% PAGE and transferred onto nitrocellulose membranes. Nitrocellulose membranes were blocked by $5 \%$ dry milk and probed overnight at $4{ }^{\circ} \mathrm{C}$ with anti-NKX2-3 antibody (Abcam, Cambridge, MA, USA), anti-PTPN2 antibody (sc-102129, Santa Cruz Biotechnology, Santa Cruz, CA), and anti-Actin antibody (Santa Cruz Biotechnology, Santa Cruz, CA) for detection.
Table 1

Genotype of PTPN2 SNP rs2542151

\begin{tabular}{lrrrl}
\hline Disease & \multicolumn{5}{l}{ CG } & \multicolumn{1}{c}{ GT } & \multicolumn{1}{c}{ TT } & $p$-value \\
\hline CD & $11(6.2 \%)$ & $56(31.6 \%)$ & $110(62.2 \%)$ & 0.0276 \\
UC & $9(4.5 \%)$ & $50(25.3 \%)$ & $139(70.2 \%)$ & 0.814 \\
IBD & $20(5.3 \%)$ & $106(28.3 \%)$ & $249(66.4 \%)$ & 0.143 \\
Control & $14(3 \%)$ & $127(27.3 \%)$ & $325(69.7 \%)$ & \\
\hline
\end{tabular}

Genotype of PTPN2 SNP rs2542152

\begin{tabular}{llccl}
$\mathrm{b}$ & \multicolumn{5}{l}{} \\
\hline Disease & \multicolumn{1}{c}{$\mathrm{CC}$} & $\mathrm{CT}$ & $\mathrm{TT}$ & $p$-value \\
\hline $\mathrm{CD}$ & $16(9.1 \%)$ & $91(52 \%)$ & $68(38.9 \%)$ & 0.00305 \\
$\mathrm{UC}$ & $38(20.3 \%)$ & $74(39.6 \%)$ & $75(40.1 \%)$ & 0.604 \\
IBD & $54(14.9 \%)$ & $165(45.6 \%)$ & $143(39.5 \%)$ & 0.178 \\
Control & $89(19.9 \%)$ & $195(43.6 \%)$ & $163(36.5 \%)$ & \\
\hline
\end{tabular}

\subsection{Statistical analyses}

Cochran-Mantel-Haenszel Statistics was used for genotype analysis. To evaluate associations with different traits, two-sided $p$-values were calculated according to Fisher using contingency tables. $p<0.05$ was considered significant.

The non-normal distributions of the paired expressions (RT-PCR results) are statistically analyzed using a one sample resampling-based permutation test using 500,000 permutations. The analysis was performed using $\mathrm{R}$ (version 2.12.0) and the onet.permutation function in the R library DAAG (version 1.03).

\section{Results}

\subsection{Genetic association of PTPN2 SNPs rs2542151 and rs2542152 with IBD}

We analyzed the rs2542151 variation in 375 IBD patients (177 CD and 198 UC) and 466 healthy controls from a familial IBD registry in central Pennsylvania. rs2542151 was found to be significantly associated with CD (Table 1a). A significant difference between the homozygous variant genotype (GG) compared to the wild type sequence (TT) was observed between CD and healthy controls $(p<0.05)$. rs 2542152 was not associated with UC $(p>0.05)$. These results are consistent with recent association studies [2-5].

We next analyzed another SNP, rs2542152, located $2.5 \mathrm{~kb}$ upstream of PTPN2, in 362 IBD patients (175 CD and $187 \mathrm{UC}$ ) and 447 healthy controls. As shown in Table 1b, rs2542152 was found to be significantly associated with CD. A significant difference between the homozygous variant genotype (CC) com- 
a

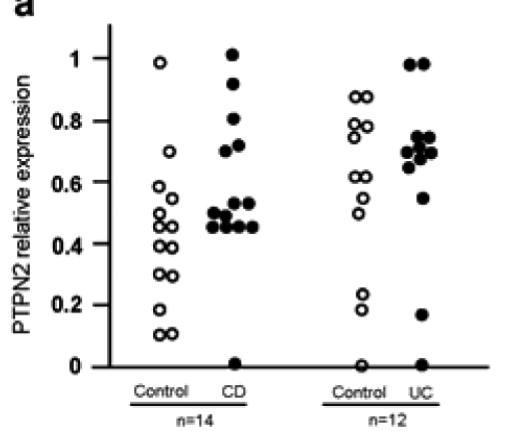

b

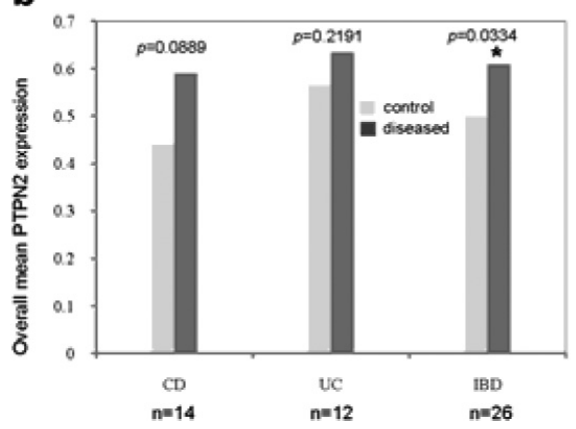

C

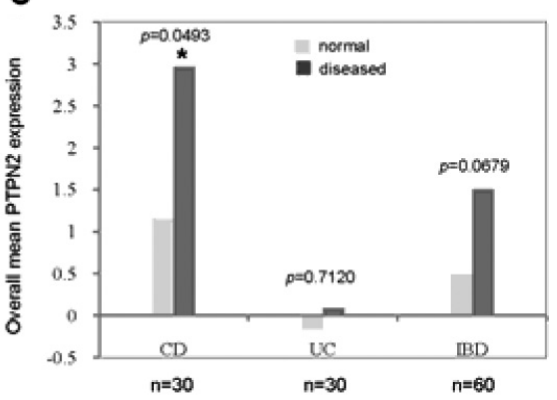

Fig. 1. PTPN2 mRNA expression in IBD. (A) Relative mRNA expression levels of PTPN2 in B cells from 14 CD and 12 UC patients with their non-IBD family member controls. RT-PCR product bands on the photograph were scanned by densitometry. Relative mRNA expression level was expressed as PTPN2 compared to GAPDH. (B) Overall mRNA expression levels of PTPN2 in CD, UC, and IBD patients compared to their non-IBD family member controls. Data are presented as the means $(* p<0.05)$. (C) mRNA expression levels of PTPN2 in diseased and adjacent normal intestinal tissues from $30 \mathrm{CD}$ and $30 \mathrm{UC}$ patients were examined by RT-PCR. Relative mRNA expression level was expressed as PTPN2 compared to GAPDH. Overall mRNA expression levels of PTPN2 in intestinal tissues from CD, UC, and IBD patients compared to adjacent normal tissues are shown. Data are presented as the mean fold changes $\left({ }^{*} p<0.05\right)$.

pared to the wild type sequence (TT) was observed between CD and all individuals without IBD $(p<0.005)$. SNP rs2542152 demonstrated a protective effect on IBD since the frequency of homozygous polymorphism (CC) in the CD patients $(9.1 \%)$ was lower than in the controls (19.9\%) (Table 1b). rs2542152 was not associated with UC $(p>0.05)$.

\subsection{Expression of PTPN2 in B cells and intestinal tissues from IBD patients}

Two studies have reported increased PTPN2 expression in intestinal biopsies from CD patients [8,9]. We examined the mRNA expression levels of PTPN2 in 14 $\mathrm{CD}$ and $12 \mathrm{UC}$ patients together with their non-IBD family members from EBV-transformed B cell lines in an IBD family registry. A total of 14 pairs were examined, and mRNA expression levels of PTPN2 from 9 pairs $(64.3 \%)$ were increased in the $\mathrm{CD}$ individual compared with the non-IBD controls However, the mRNA expression level of PTPN2 was not significantly altered between 12 UC and their non-IBD family mem- bers (Fig. 1A). The overall mean PTPN2 / GAPDH expression values with the standard errors (SD) are shown in Fig. 1B: $0.593 \pm 0.247$ (CD) vs. $0.443 \pm 0.246$ (non-IBD control) $(p=0.0889) ; 0.635 \pm 0.273$ (UC) vs. $0.565 \pm 0.278$ (non-IBD control) $(p=0.2191)$; and $0.613 \pm 0.255$ (IBD) vs. $0.499 \pm 0.263$ (non-IBD control) $(p=0.0334)$.

Next we examined the mRNA expression of PTPN2 in intestinal tissues. Diseased intestinal and adjacent non-diseased samples obtained from $30 \mathrm{CD}$ and $30 \mathrm{UC}$ patients were used for this study. As shown in Fig. 1C, the mRNA expression level of PTPN2 was increased in the diseased intestinal tissues compared with adjacent non-diseased tissues from $30 \mathrm{CD}$ patients. mRNA expression levels of PTPN2 from 19 CD patients (63.3\%) were increased in the diseased tissues compared with adjacent non-diseased tissues. However, the mRNA expression level of PTPN2 was not significantly altered between the diseased intestinal tissues and adjacent non-diseased tissues from 30 UC patients (Fig. 1C). The overall mean PTPN2 / GAPDH expression fold changes with SD are: $2.97 \pm 6(\mathrm{CD})$ vs. $1.15 \pm 4.6$ 
a

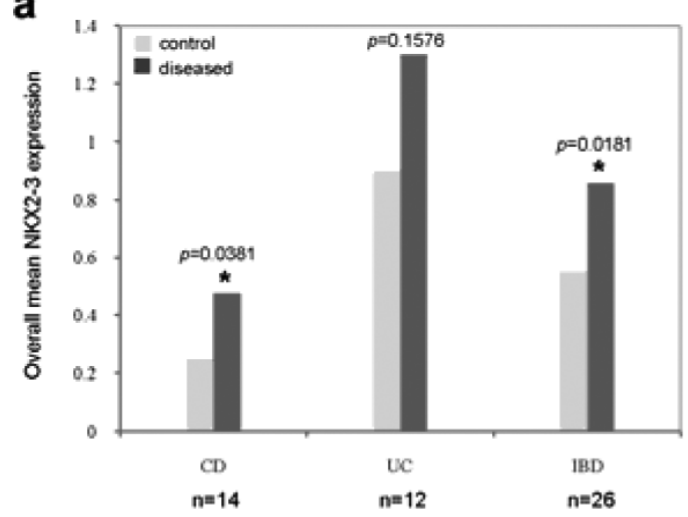

b

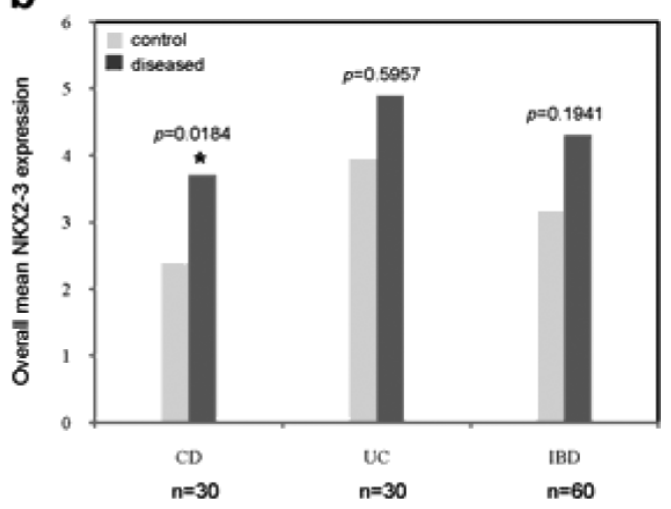

Fig. 2. NKX2-3 mRNA expression in IBD. (A) mRNA expression levels of NKX2-3 in B cells from 14 CD and 12 UC patients with their non-IBD family member controls. The relative mRNA expression level was expressed as NKX2-3 compared to GAPDH. Overall mRNA expression levels of NKX2-3 in CD, UC, and IBD patients compared to their non-IBD family member controls are shown. Data are presented as the means $(* p<$ 0.05). (B) mRNA expression levels of NKX2-3 in diseased and adjacent normal intestinal tissues from $30 \mathrm{CD}$ and $30 \mathrm{UC}$ patients. The relative mRNA expression level was expressed as NKX2-3 compared to GAPDH. Overall mRNA expression levels of NKX2-3 in intestinal tissues from $\mathrm{CD}, \mathrm{UC}$, and IBD patients compared to adjacent normal tissues are shown. Data are presented as the means $\left({ }^{*} p<0.05\right)$.

(non-diseased tissues) $(p=0.0493) ; 0.09 \pm 5.82(\mathrm{UC})$ vs. $-0.16 \pm 4.36$ (non-diseased tissues) $(p=0.7120)$; and $1.5 \pm 6.04$ (IBD) vs. $0.49 \pm 4.49$ (non-diseased tissues) $(p=0.0679)$.

Taken together, mRNA expression levels of PTPN2 were significantly increased in intestinal tissues (CD: normal $=2.58, p=0.0493$ ), and nearly significantly increased in B cells $(\mathrm{CD}$ : normal $=1.34, p=0.0889)$ from $\mathrm{CD}$ patients. However, mRNA expression levels of PTPN2 were not significantly increased in UC.

\subsection{Expression of NKX2-3 in B cells and intestinal tissues from IBD patients}

Transcription factor NKX2-3 is associated with IBD and its expression is up-regulated in CD patients [10]. To confirm these results, we first examined NKX2-3 mRNA expression levels in $14 \mathrm{CD}$ and $12 \mathrm{UC}$ together with their non-IBD family members from $\mathrm{B}$ cell lines as indicated above in the PTPN2 study. As shown in Fig. 2A, the mRNA expression level of NKX2-3 was significantly increased in B cells in CD and IBD (CD and UC combined) compared with non-IBD family member controls, but not significantly increased in UC. The overall mean NKX2-3 / GAPDH expression values with SD are: $0.477 \pm 0.287(\mathrm{CD})$ vs. $0.252 \pm 0.278$ (non-IBD control) $(p=0.0381) ; 1.3 \pm 0.807$ (UC) vs. $0.898 \pm 0.646$ (non-IBD control) $(p=0.1576)$; and $0.858 \pm 0.779$ (IBD) vs. $0.55 \pm 0.656$ (non-IBD control) $(p=0.0181)$.

Next we examined the mRNA expression of NKX23 in intestinal tissues. Diseased intestinal and adjacent non-diseased samples obtained from $30 \mathrm{CD}$ and $30 \mathrm{UC}$ patients as indicated above in the PTPN2 study were used for this study. As shown in Fig. 2B, the mRNA expression level of NKX2-3 was increased in the diseased intestinal tissues compared with adjacent nondiseased tissues from $30 \mathrm{CD}$ patients, but not increased in 30 UC patients or total 60 IBD patients. The overall mean NKX2-3 / GAPDH expression values with SD are: $3.71 \pm 3.4(\mathrm{CD})$ vs. $2.38 \pm 1.59$ (non-diseased tissues) $(p=0.0184) ; 4.89 \pm 6.32$ (UC) vs. $3.95 \pm$ 7.02 (non-diseased tissues) $(p=0.5957)$; and $4.3 \pm$ 5.07 (IBD) vs. $3.16 \pm 5.11$ (non-diseased tissues) ( $p=$ $0.1941)$.

\subsection{Down-regulation of PTPN2 in NKX2-3 shRNA knockdown human cells}

We found that PTPN2 was down-regulated by NKX2-3 knockdown in various human cells using cDNA microarray [11-13]. The microarray ratios of PTPN2 (knockdown vs. control) were shown in Fig. 3A: 0.63 in a B cell line from a CD patient, 0.93 in a $B$ cell line from a UC patient, and 0.77 in a HIMEC line. To validate microarray results, we examined mRNA and protein expression of PTPN2 in NKX2-3 knockdown cells. mRNA and protein expression levels of PTPN2 were significantly decreased by NKX2-3 knockdown in a $\mathrm{B}$ cell line from a CD patient, a B cell line from a UC patient, and a HIMEC line (Fig. 3B and $3 \mathrm{C})$. 


\begin{tabular}{lcc}
\hline Cell type & Array ratio & $p$-value \\
\hline B cell $(C D)$ & 0.63 & 0.0038 \\
B cell (UC) & 0.93 & 0.0215 \\
HIMEC & 0.77 & 0.0522 \\
\hline
\end{tabular}

a
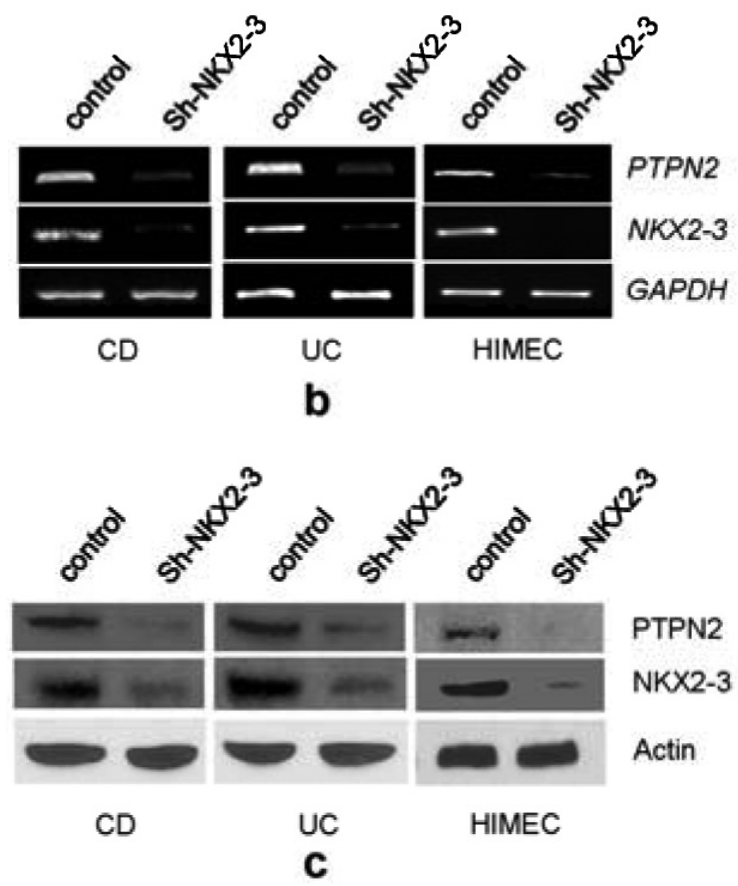

Fig. 3. PTPN2 is down-regulated by NKX2-3 knockdown. (A) cDNA microarray ratio of PTPN2 (knockdown vs. control) in NKX2-3 knockdown from a B cell line from a CD patient, a B cell line from a UC patient, and a HIMEC line. (B) RT-PCR analysis of mRNA expression levels of PTPN2 and NKX2-3 in NKX2-3 knockdown from a $B$ cell line from a $C D$ patient, a B cell line from a UC patient, and a HIMEC line. GAPDH is loading control. (C) Western Blotting analysis of protein expression levels of PTPN2 and NKX2-3 in NKX2-3 knockdown from a B cell line from a CD patient, a B cell line from a UC patient, and a HIMEC line. Actin is loading control.

\subsection{Positive correlation between gene expression of NKX2-3 and PTPN2 in IBD patients}

Since both NKX2-3 and PTPN2 are up-regulated in CD patients and PTPN2 is down-regulated by NKX23 knockdown in human cells (Fig. 3), we next examined whether NKX2-3 expression was correlated with PTPN2 expression in IBD patients. First we examined mRNA expression levels of NKX2-3 and PTPN2 in B cells from $14 \mathrm{CD}$ and $12 \mathrm{UC}$ patients. Analysis showed a positive correlation between expression of NKX2-3 and PTPN2 for CD $(r=0.6023, p<0.001)$ and for UC $(r=0.5928, p<0.005)$ (Fig. 4A). There was also a positive correlation between expression of NKX2-3 and PTPN2 for both CD and UC $(n=26)(r=0.4936$, $p<0.01$ ) (Fig. 4A).

Second, we examined mRNA expression levels of NKX2-3 and PTPN2 in diseased intestinal tissues from $30 \mathrm{CD}$ and $30 \mathrm{UC}$ patients. Analysis showed a positive correlation between expression of NKX2-3 and PTPN2 for CD $(r=0.8272, p<0.0001)$ and for UC $(r=$ $0.6355, p<0.0001$ ) (Fig. 4B). There was also a positive correlation between expression of NKX2-3 and PTPN2 for both CD and UC $(n=60)(r=0.5485, p<0.0001)$ (Fig. 4B).

\section{Discussion}

rs2542151, located $5.5 \mathrm{~kb}$ upstream of PTPN2, was associated with CD, type T1D, and RA [2]. We not only replicated an association of rs2542151 with CD using a familial IBD registry from central Pennsylvania, but also were able to show a novel association of rs 2542152 (located $2.5 \mathrm{~kb}$ upstream of PTPN2) with CD. We demonstrated that mRNA expression of PTPN2 was increased in B cells and intestinal tissues from CD patients, but not UC patients. Furthermore, expression of PTPN2 was regulated by NKX2-3 in IBD.

The association of rs 2542151 with $\mathrm{CD}$ was reported by several studies in European population [25]. In this study, we replicated the association in a small IBD patient population from Central Pennsylvania area. Historically it was mainly populated by immigrants from the United Kingdom and Western Europe (Germany, the Netherlands), and presents as having a patient population with a relatively homogeneous genetic background. The rs 2542152 polymorphism was associated with a significantly relative risk reduction of developing IBD. The mechanism of the protective effect on IBD by rs 2542152 polymorphism is unclear. Since the rs 2542152 polymorphisms is located within a binding motif of several transcription factors, such as TBP, FOXP3, POU2F2, POU3F2, HOXD8, C/EBP and AGL3 (predicated by PROMO software: http://alggen.lsi.upc.es/cgibin/promo_v3/promo/promoinit.cgi?dirDB=TF_8.3), it could affect PTPN2 promoter activity or gene expression. However, not all diseases are caused by altered gene expression levels. Thus analyzing the effect of SNPs on gene expression levels will not always explain the mechanism by which disease-associated SNP alleles cause the phenotypic changes.

Protein tyrosine phosphatases play critical roles in regulating cell proliferation, differentiation and sur- 
a
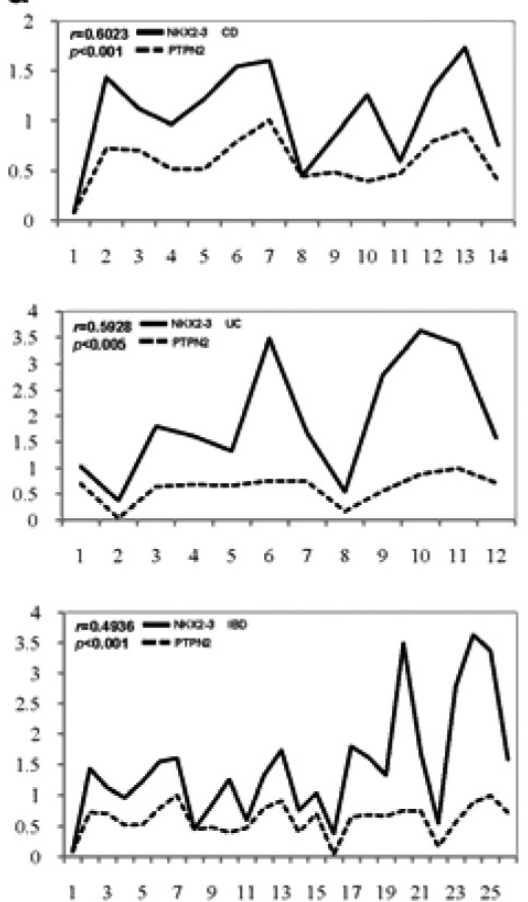

b
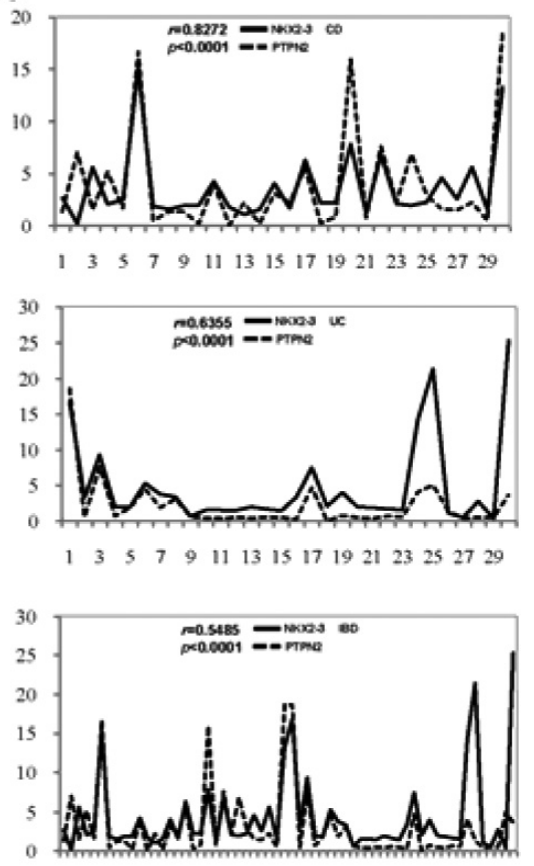

1471013161922252831343740434649525558

Fig. 4. The correlation between the expression levels of NKX2-3 and PTPN2 in B cells and intestinal tissues from IBD patients. (A) The correlation between the expression levels of NKX2-3 and PTPN2 in B cells from 14 CD (upper), 12 UC (middle), and 26 IBD patients (bottom). RT-PCR results were normalized by GAPDH in every patient. Correlation coefficient $(r)$ and $p$ value are shown. (B) The correlation between the expression levels of NKX2-3 and PTPN2 in diseased intestinal tissues from $30 \mathrm{CD}$ (upper), $30 \mathrm{UC}$ (middle), and 60 IBD patients (bottom). RT-PCR results were normalized by GAPDH in every patient. Correlation coefficient $(r)$ and $p$ value are shown.

vival [6]. In intestinal cells, PTPN2 regulates epithelial permeability and is a key negative regulator of important immune mediators STAT1 and STAT3, as well as p38 and ERK1/2 phosphorylation. Consequently, it attenuates both IFN $\gamma$ and TNF $\alpha$ induced signaling and release of proinflammatory cytokines [7]. As supported by the phenotype of the PTPN2 $2^{-/}$mouse, PTPN2 is a multiple regulator of the immune system, affecting hematopoiesis in several lineages and controlling systemic inflammatory responses [17]. PTPN2 ${ }^{-/-}$mice have high expression of several cytokines associated with CD, such as IFN- $\gamma$, TNF- $\alpha$, and IL-12 [7,18]. $\mathrm{TNF}-\alpha$, for example, is an important effector of CD in humans, and anti-TNF- $\alpha$ therapy has proven to be an effective treatment modality in patients [19]. Additionally, the onset of IBD such as CD in humans has been described as an outcome of a combination of Toll-like receptor-4 (TLR4)-mediated LPS signaling and IFN- $\gamma$ production by Th1 cells, resulting in NO production contributing to the pathogenesis of the gut [20].

To investigate the mechanism of PTPN2 in IBD pathogenesis, we studied mRNA expression of this gene. Expression levels of PTPN2 have not been well investigated in IBD. Two studies showed the increased expression of PTPN2 in CD [8,9]. Our results demonstrated that expression of PTPN2 was up-regulated in both $\mathrm{B}$ cells and intestinal tissues from CD patients, but its expression was not significantly altered in UC patients. Since our studies showed rs2542151 and rs2542152 were associated with CD but not UC, there may be a possible connection between the association of the two SNPs with PTPN2 up-regulation in CD. NKX2-3 is associated with IBD and up-regulated in $\mathrm{CD}$ patients [10]. We found that expression of NKX2-3 was increased in B cells and intestinal tissues from CD patients, and expression of PTPN2 was decreased in NKX2-3 knockdown human cells. These results suggested that there might be close correlation between PTPN2 and NKX2-3 expression in IBD patients. Indeed, we found a significantly positive correlation between PTPN2 and NKX2-3 expression in IBD patients both in B cells and in intestinal tissues. The mechanism of the regulation of PTPN2 by NKX2-3 is presently unclear.

PTPN2 is involved in T-cell proliferation [21] and B cell development [22]. During development, NKX2- 
3 is expressed in midgut and hindgut mesoderm and spleen, as well as in pharyngeal endoderm [23,24]. NKX2-3 plays an important role in normal B cell development and $\mathrm{T}$ cell dependent immune response [25]. PTPN2 is activated by IFN $\gamma$ and regulates intestinal epithelial barrier function [9]. PTPN2 is also up-regulated by TNF- $\alpha$ in intestinal epithelial cells [8]. Both of these cytokines play important roles in the pathogenesis of IBD, and of CD in particular. TNF- $\alpha$ causes cytoplasmic accumulation of PTPN2 in intestinal epithelial cells. Our previous cDNA microarray data (unpublished) showed that both IFN $\gamma$ and TNF- $\alpha$ were down-regulated by NKX2-3 knockdown in B cells. It is possible that PTPN2 could be indirectly regulated by NKX2-3 via TNF- $\alpha$ or IFN $\gamma$ pathways. Further studies are needed to investigate the mechanism by which NKX2-3 regulates PTPN2 expression. The functional role that PTPN2 might play in the etiology of intestinal inflammation and IBD remains to be fully defined. As a whole, these data suggest PTPN2 may have an important role in $\mathrm{CD}$ pathogenesis and may represent a potential therapeutic target for IBD.

\section{Acknowledgments}

This work was supported by a grant from the Philadelphia Health Care Trust (WAK), the Carlino fund for IBD research at the Milton S. Hershey Medical Center (WAK), Penn State College of Medicine, and the Milton S. Hershey Penn State College of Medicine Surgery Research Feasibility Grant (WY).

\section{References}

[1] J.H. Cho, The genetics and immunopathogenesis of inflammatory bowel disease, Nat Rev Immunol 8 (2008), 458-466.

[2] WTCCC, Genome-wide association study of 14,000 cases of seven common diseases and 3,000 shared controls. Nature 447 (2007), 661-678.

[3] M. Parkes, J.C. Barrett, N.J. Prescott, M. Tremelling, C.A. Anderson, S.A. Fisher, R.G. Roberts, E.R. Nimmo, F.R. Cummings, D. Soars, H. Drummond, C.W. Lees, S.A. Khawaja, R. Bagnall, D.A. Burke, C.E. Todhunter, T. Ahmad, C.M. Onnie, W. McArdle, D. Strachan, G. Bethel, C. Bryan, C.M. Lewis, P. Deloukas, A. Forbes, J. Sanderson, D.P. Jewell, J. Satsangi, J.C. Mansfield, L. Cardon and C.G. Mathew, Sequence variants in the autophagy gene IRGM and multiple other replicating loci contribute to Crohn's disease susceptibility, Nat Genet 39 (2007), 830-832.

[4] A. Franke, T. Balschun, T.H. Karlsen, J. Hedderich, S. May, T. Lu, D. Schuldt, S. Nikolaus, P. Rosenstiel, M. Krawczak and S. Schreiber, Replication of signals from recent studies of Crohn's disease identifies previously unknown disease loci for ulcerative colitis, Nat Genet 40 (2008), 713-715.
[5] R.K. Weersma, P.C. Stokkers, I. Cleynen, S.C. Wolfkamp, L. Henckaerts, S. Schreiber, G. Dijkstra, A. Franke, I.M. Nolte, P. Rutgeerts, C. Wijmenga and S. Vermeire, Confirmation of multiple Crohn's disease susceptibility loci in a large DutchBelgian cohort, Am J Gastroenterol 104 (2009), 630-638.

[6] N.K. Tonks and B.G. Neel, Combinatorial control of the specificity of protein tyrosine phosphatases, Curr Opin Cell Biol 13 (2001), 182-195.

[7] K.M. Heinonen, F.P. Nestel, E.W. Newell, G. Charette, T.A. Seemayer, M.L. Tremblay and W.S. Lapp, T-cell protein tyrosine phosphatase deletion results in progressive systemic inflammatory disease, Blood 103 (2004), 3457-3464.

[8] M. Scharl, D.F. McCole, A. Weber, S.R. Vavricka, P. Frei, S. Kellermeier, T. Pesch, M. Fried and G. Rogler, Protein tyrosine phosphatase N2 regulates TNFalpha-induced signalling and cytokine secretion in human intestinal epithelial cells, Gut 60 (2011), 189-197.

[9] M. Scharl, G. Paul, A. Weber, B.C. Jung, M.J. Docherty, M. Hausmann, G. Rogler, K.E. Barrett and D.F. McCole, Protection of epithelial barrier function by the Crohn's disease associated gene protein tyrosine phosphatase n2, Gastroenterology 137 (2009), 2030-2040 e5.

[10] W. Yu, Z. Lin, A.A. Kelly, J.P. Hegarty, L.S. Poritz, Y. Wang, T. Li, S. Schreiber and W.A. Koltun, Association of a Nkx2-3 polymorphism with Crohn's disease and expression of Nkx23 is up-regulated in B cell lines and intestinal tissues with Crohn's disease, Journal of Crohn's and Colitis 3 (2009), 189-195.

[11] W. Yu, Z. Lin, J.P. Hegarty, G. John, X. Chen, P.W. Faber, A.A. Kelly, Y. Wang, L.S. Poritz, S. Schreiber and W.A. Koltun, Genes regulated by Nkx2-3 in siRNA-mediated knockdown B cells: implication of endothelin-1 in inflammatory bowel disease, Mol Genet Metab 100 (2010), 88-95.

[12] W. Yu, Z. Lin, J.P. Hegarty, X. Chen, A.A. Kelly, Y. Wang, L.S. Poritz and W.A. Koltun, Genes differentially regulated by NKX2-3 in B cells between ulcerative colitis and Crohn's disease patients and possible involvement of EGR1, Inflammation (2011), (accepted, in press).

[13] W. Yu, J.P. Hegarty, A. Berg, X. Chen, G. West, A.A. Kelly, Y. Wang, L.S. Poritz, W.A. Koltun and Z. Lin, NKX2-3 Transcriptional Regulation of Endothelin-1 and VEGF Signaling in Human Intestinal Microvascular Endothelial Cells, PLoS One 6 (2011), e20454.

[14] G. John, J.P. Hegarty, W. Yu, A. Berg, D.M. Pastor, A.A. Kelly, Y. Wang, L.S. Poritz, S. Schreiber, W.A. Koltun and Z. Lin, NKX2-3 variant rs11190140 is associated with IBD and alters binding of NFAT, Mol Genet Metab 104 (2011), 174-179.

[15] Z. Lin, G. John, J.P. Hegarty, A. Berg, W. Yu, Y. Wang, A.A. Kelly, B.Z. Peterson, L.S. Poritz, J. Floros and W.A. Koltun, Genetic Variants and Monoallelic Expression of Surfactant Protein-D in Inflammatory Bowel Disease, Ann Hum Genet 75 (2011), 559-568.

[16] W. Yu, Z. Lin, D.M. Pastor, J.P. Hegarty, X. Chen, A.A. Kelly, Y. Wang, L.S. Poritz and W.A. Koltun, Genes Regulated by Nkx2-3 in Sporadic and Inflammatory Bowel DiseaseAssociated Colorectal Cancer Cell Lines, Dig Dis Sci 55 (2010), 3171-3180.

[17] K.M. Doody, A. Bourdeau and M.L. Tremblay, T-cell protein tyrosine phosphatase is a key regulator in immune cell signaling: lessons from the knockout mouse model and implications in human disease, Immunol Rev 228 (2009), 325-341.

[18] O.H. Nielsen, I. Kirman, N. Rudiger, J. Hendel and B. Vainer, Upregulation of interleukin-12 and -17 in active inflammatory bowel disease, Scand J Gastroenterol 38 (2003), 180-185. 
[19] M.T. Osterman and G.R. Lichtenstein, Current and Future Anti-TNF Therapy for Inflammatory Bowel Disease, Curr Treat Options Gastroenterol 10 (2007), 195-207.

[20] D. Shi, J. Das and G. Das, Inflammatory bowel disease requires the interplay between innate and adaptive immune signals, Cell Res 16 (2006), 70-74.

[21] M. Dupuis, M. De Jesus Ibarra-Sanchez, M.L. Tremblay and P. Duplay, Gr-1+ myeloid cells lacking T cell protein tyrosine phosphatase inhibit lymphocyte proliferation by an IFNgamma- and nitric oxide-dependent mechanism, J Immunol 171 (2003), 726-732.

[22] A. Bourdeau, N. Dube, K.M. Heinonen, J.F. Theberge, K.M Doody and M.L. Tremblay, TC-PTP-deficient bone marrow stromal cells fail to support normal B lymphopoiesis due to abnormal secretion of interferon- $\{$ gamma $\}$, Blood $109(2007)$, 4220-4228.
[23] Y. Fu, W. Yan, T.J. Mohun and S.M. Evans, Vertebrate tinman homologues XNkx2-3 and XNkx2-5 are required for heart formation in a functionally redundant manner, Development 125 (1998), 4439-4449.

[24] O. Pabst, A. Schneider, T. Brand and H.H. Arnold, The mouse Nkx2-3 homeodomain gene is expressed in gut mesenchyme during pre- and postnatal mouse development, Dev Dyn 209 (1997), 29-35.

[25] D. Tarlinton, A. Light, D. Metcalf, R.P. Harvey and L. Robb, Architectural defects in the spleens of Nkx2-3-deficient mice are intrinsic and associated with defects in both B cell maturation and $\mathrm{T}$ cell-dependent immune responses, J Immunol $\mathbf{1 7 0}$ (2003), 4002-4010. 


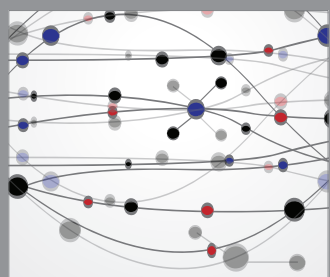

The Scientific World Journal
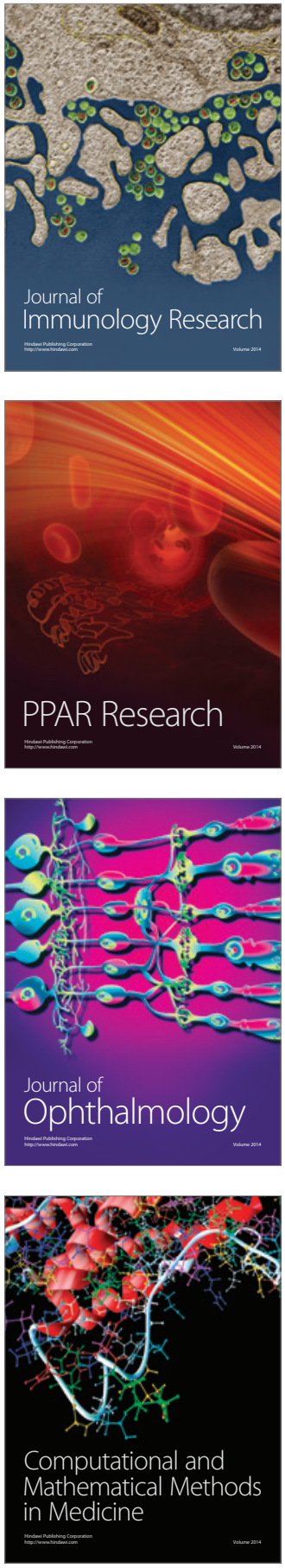

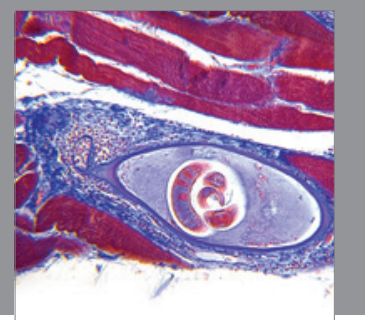

Gastroenterology

Research and Practice
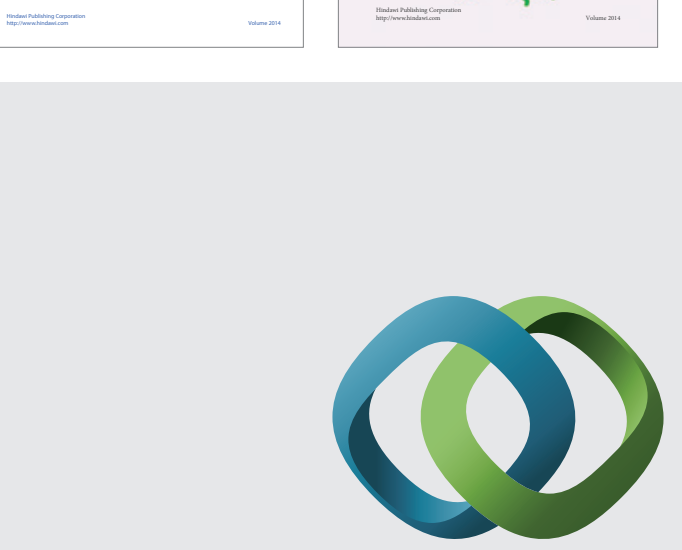

\section{Hindawi}

Submit your manuscripts at

http://www.hindawi.com
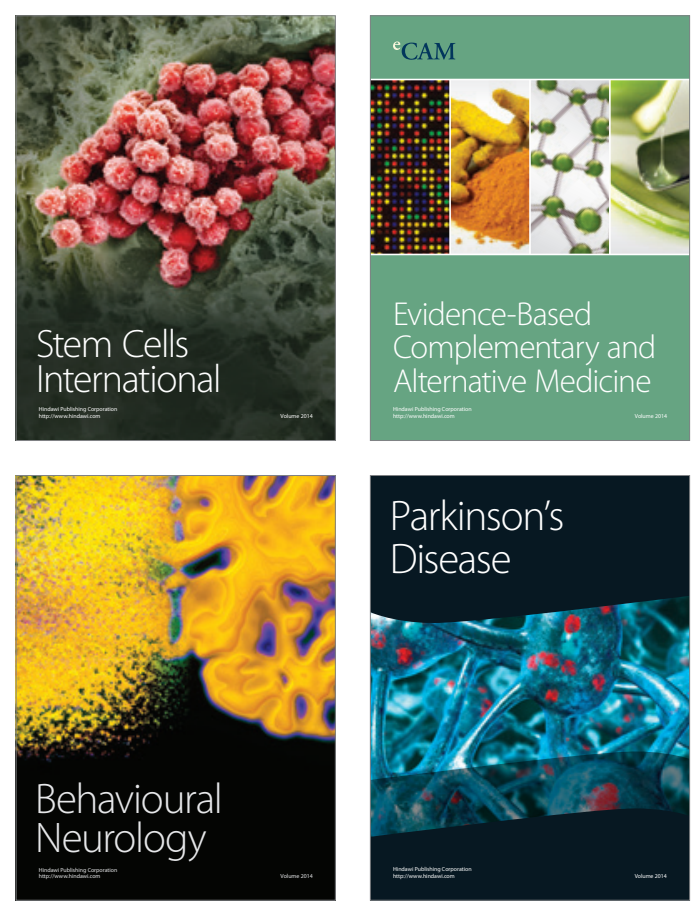

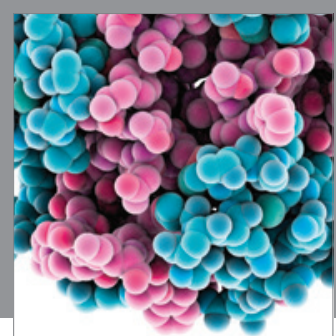

Journal of
Diabetes Research

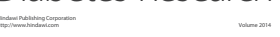

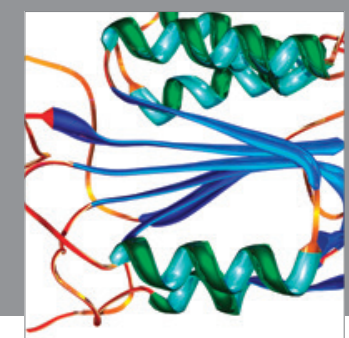

Disease Markers
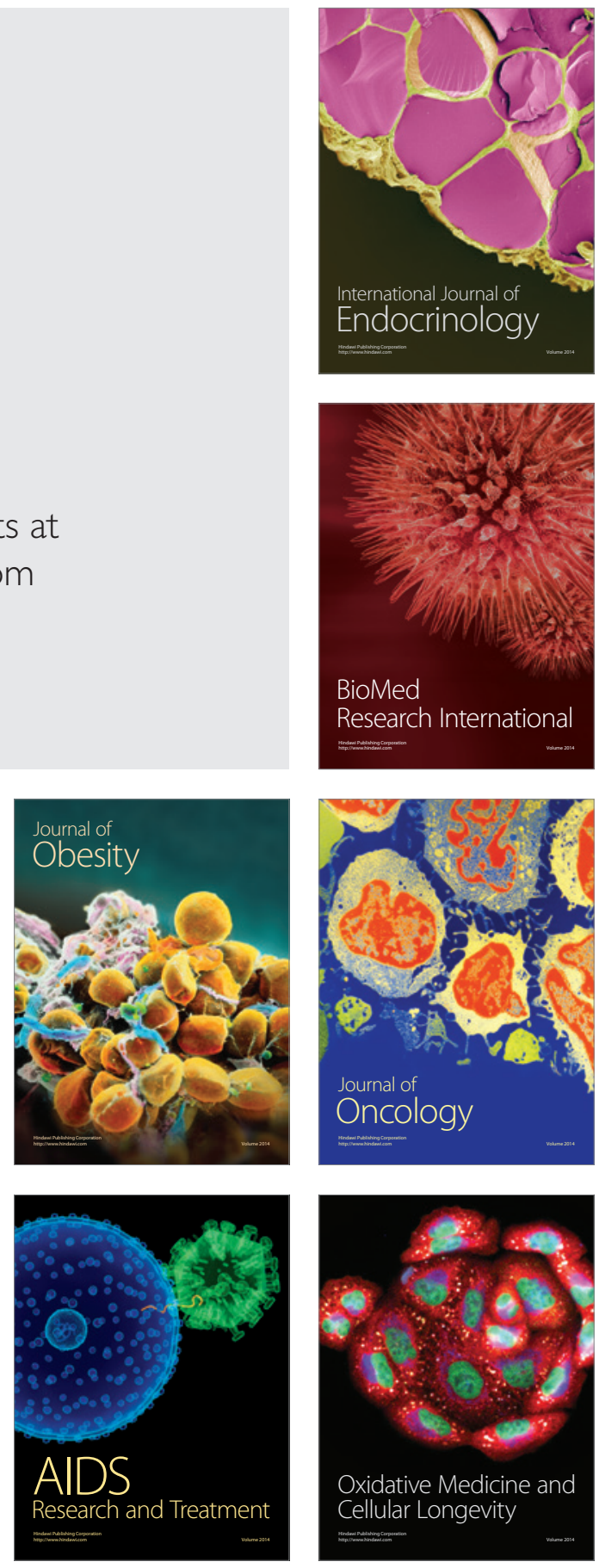